\title{
Markov Chain Analysis of Succession in a Rocky Subtidal Community
}

\author{
M. Forrest Hill, ${ }^{1, *}$ Jon D. Witman, ${ }^{2, \dagger}$ and Hal Caswell ${ }^{3, *}$
}

1. Institute of Theoretical Dynamics, University of California, Davis, California 95616;

2. Department of Ecology and Evolutionary Biology, Brown University, Providence, Rhode Island 02912;

3. Biology Department MS-34, Woods Hole Oceanographic Institution, Woods Hole, Massachusetts 02543

Submitted June 18, 2003; Accepted February 24, 2004;

Electronically published July 1, 2004

ABSTRACT: We present a Markov chain model of succession in a rocky subtidal community based on a long-term (1986-1994) study of subtidal invertebrates (14 species) at Ammen Rock Pinnacle in the Gulf of Maine. The model describes successional processes (disturbance, colonization, species persistence, and replacement), the equilibrium (stationary) community, and the rate of convergence. We described successional dynamics by species turnover rates, recurrence times, and the entropy of the transition matrix. We used perturbation analysis to quantify the response of diversity to successional rates and species removals. The equilibrium community was dominated by an encrusting sponge (Hymedesmia) and a bryozoan (Crisia eburnea). The equilibrium structure explained $98 \%$ of the variance in observed species frequencies. Dominant species have low probabilities of disturbance and high rates of colonization and persistence. On average, species turn over every 3.4 years. Recurrence times varied among species (7-268 years); rare species had the longest recurrence times. The community converged to equilibrium quickly (9.5 years), as measured by Dobrushin's coefficient of ergodicity. The largest changes in evenness would result from removal of the dominant sponge Hymedesmia. Subdominant species appear to increase evenness by slowing the dominance of Hymedesmia. Comparison of the subtidal community with intertidal and coral reef communities revealed that disturbance rates are an order of magnitude higher in coral reef than in rocky intertidal and subtidal communities. Colonization rates and turnover times, however, are lowest and longest

\footnotetext{
* Present address: ENTRIX, 590 Ygnacio Valley Road, Walnut Creek, California 94596; e-mail: fhill@entrix.com.

† E-mail: jon_witman@brown.edu.

₹ Corresponding author; e-mail: hcaswell@whoi.edu.
}

Am. Nat. 2004. Vol. 164, pp. E46-E61. (c) 2004 by The University of Chicago. 0003-0147/2004/16402-30238\$15.00. All rights reserved. in coral reefs, highest and shortest in intertidal communities, and intermediate in subtidal communities.

Keywords: Markov chain, sensitivity analysis, transition matrix, species diversity, entropy, Dobrushin's coefficient.

The dynamics of an ecological community are often described by changes in species composition over time. We use the term "succession" to refer to these changes. Succession is no longer viewed as a deterministic development toward a unique stable climax community (Connell and Slatyer 1977). Instead, it is widely recognized that disturbance, dispersal, colonization, and species interactions produce patterns and variability on a range of temporal and spatial scales.

Markov chains were introduced as models of succession by Waggoner and Stephens (1970) and Horn (1975). These models imagine the landscape as a large (usually infinite) set of patches or points in space. The state of a point is given by the list of species that occupy it. In one class of models, this list may include multiple coexisting populations; such models are called patch-occupancy models (e.g., Caswell and Cohen 1991a, 1991b). In another class of models, points are occupied by single individuals rather than populations. Such models have been applied to forests (Waggoner and Stephens 1970; Horn 1975; Runkle 1981; Masaki et al. 1992), plant communities (Isagi and Nakagoshi 1990; Aaviksoo 1995), insect assemblages (Usher 1979), coral reefs (Tanner et al. 1994, 1996), and rocky intertidal communities (Wootton 2001b, 2001c).

Successional dynamics are modeled by defining the probability distribution of the patch state at time $t+1$, conditional on its state at time $t$. This distribution may depend on time, location, and local or global state frequencies. Time-varying models can be analyzed as nonhomogeneous Markov chains (e.g., Hill et al. 2002). Models with dependence on local state frequencies are nonlinear stochastic cellular automata (e.g., Caswell and Etter 1999). Models with dependence on global state frequencies are nonlinear Markov chains (Caswell and Cohen 
1991a, 1991b; Hill 2000), which are mean-field models for the corresponding cellular automata.

Models with time-invariant transition probabilities are homogeneous, finite-state Markov chains. Here, we use such a chain to analyze succession in a rocky subtidal community. A detailed statistical analysis of the model that examined the effects of spatial and temporal variation showed that although statistically significant, the effects are so small as to be biologically trivial, regardless of whether asymptotic or transient properties of the community are examined (Hill et al. 2002). We will explore the effects of nonlinearity elsewhere.

Suppose that there are $s$ possible states. Let $X(t) \in$ $\{1,2, \ldots, s\}$ denote the state of a point at time $t$. The model is defined by a transition matrix $\mathbf{P}$, whose elements, $p_{i j}$, are the conditional probabilities:

$$
p_{i j}=P[X(t+1)=i \mid X(t)=j] \quad i, j=1, \ldots, s .
$$

$\mathbf{P}$ is column-stochastic (i.e., each column sums to 1 ). Let $\mathbf{x}$ be a probability vector (i.e., $x_{i} \geq 0, \sum_{i} x_{i}=1$ ) whose entries give the probability that a point is in state $i$. Then

$$
\mathbf{x}(t+1)=\mathbf{P} \mathbf{x}(t) .
$$

If the community is conceived of as an ensemble of points, each experiencing an independent realization of the stochastic process given by $\mathbf{P}$, then $\mathbf{x}(\mathrm{t})$ defines the state of the community; its entries are the expected relative frequencies of point states at time $t$.

We believe that Markov chain models are a valuable but underutilized tool in community ecology. Consequently, one of our goals is to extend the analysis of community dynamics by a more systematic exploration of the properties of Markov chain models than has appeared in any previous study. This is the first time that some of these results have been reported for any community. To help interpret them, we will contrast our results with those obtained from matrices reported by Wootton (2001c) for intertidal communities in Washington and by Tanner et al. (1994) for coral reefs in Australia. Such comparative analysis will become more powerful and more valuable as additional data sets are collected (see Wootton 2001a for a comparison along different lines from ours).

\section{The Subtidal Community}

Rocky subtidal habitats extend from the intertidal area down to the upper limit of the deep sea at about $200 \mathrm{~m}$ (Witman and Dayton 2001). Here we will study a subtidal community on vertical rock walls; such communities are dominated by epifaunal invertebrates (Sebens 1986). They are subject to lower disturbance rates than communities on horizontal substrates at similar depths. Witman and Dayton (2001) have reviewed the processes (disturbance, predation, and competition) operating in these communities. Because sessile organisms require substrate, competition for space plays a key role; disturbance and predation open up space for colonization. Colonization usually requires recruitment of larvae. At shallow subtidal depths, larvae sometimes settle in dense aggregations that completely cover available space, but this seems to happen less often at deeper depths, including the depths from which our data come (Witman and Dayton 2001).

Subtidal succession has typically been studied on small $\left(<0.1 \mathrm{~m}^{2}\right)$ artificial substrata such as plates or tiles (Sutherland and Karlson 1977; Osman 1982), so there are few studies of succession on natural hard substrates for comparison. Keough (1984) found that the dynamics of sessile epifauna on fan-shaped shells of a large bivalve were dominated by recruitment, with high species turnover as residents died. Disturbance was low in these small, discrete habitats. Physical disturbance had a greater influence on epifaunal communities on shallow (4-12-m depth) rock walls in the Caribbean, where the communities were impacted three times in six years by hurricanes, which created small patches $\left(<25 \mathrm{~cm}^{2}\right)$ for succession (Witman 1992). Since wave energy dissipates with depth, natural physical disturbance should decrease with depth in the rocky subtidal community (Witman and Dayton 2001).

\section{Parameter Estimation}

Our analysis focuses on a subtidal rock wall community at 30-35-m depth on Ammen Rock Pinnacle in the central Gulf of Maine (Witman and Sebens 1988; Leichter and Witman 1997). Nine permanently marked $0.25-\mathrm{m}^{2}$ quadrats, positioned horizontally along a $20-\mathrm{m}$ span of the rock wall habitat, were photographed each year with a Nikonos camera mounted on a quadrapod frame (Witman 1985). A total of 14 species of sponges, sea anemones, ascidians, bryozoans, and polychaetes were identified in the photos (table 1). For reasons that will become apparent, we have ordered the species in decreasing rank order of dominance in the equilibrium community.

Transition frequencies were measured by superimposing a rectangular lattice of $30 \times 20$ points at $1-\mathrm{cm}$ intervals onto $31.25 \times 20$-cm color prints made from highresolution color slides of the quadrat photos. This corresponds to approximately a $2-\mathrm{cm}$ interval on the actual substrate $\left([0.25 / 0.06]^{1 / 2}=2.04\right)$, which approximates the size of the smallest organisms in the data set. At each time, the lattice was aligned to assure that species were always censused at the same points. We recorded the species occupying each point on each quadrat in each year, for a total of $\sim 42,000$ points (the species occupying 
Table 1: Subtidal species found in quadrats at 30-m depth on Ammen Rock Pinnacle in the Gulf of Maine

\begin{tabular}{lllr}
\hline Model states & Species type & State ID & Number \\
\hline Hymedesmia sp. 1 & Sponge & HY1 & 14,875 \\
Crisia eburnea & Bryozoan & CRI & 9,915 \\
Myxilla fimbriata & Sponge & MYX & 4,525 \\
Mycale lingua & Sponge & MYC & 3,001 \\
Filograna implexa & Polychaete & FIL & 2,219 \\
Urticina crassicornis & Sea anemone & URT & 992 \\
Ascidia callosa & Ascidian & ASC & 1,052 \\
Aplidium pallidum & Ascidian & APL & 1,166 \\
Hymedesmia sp. 2 & Sponge & HY2 & 1,226 \\
Idmidronea atlantica & Bryozoan & IDM & 730 \\
Coralline Algae & Encrusting algae & COR & 875 \\
Metridium senile & Sea anemone & MET & 1,298 \\
Parasmittina jeffreysi & Bryozoan & PAR & 402 \\
Spirorbis spirorbis & Polychaete & SPI & 225 \\
Bare rock & & BR & 4,266 \\
\hline
\end{tabular}

Note: Species are identified in the model using the state codes listed under State ID. Number of points counted per species is shown in the right-hand column of the table. Species are listed in decreasing order of abundance in the stationary community.

a few points were unidentifiable; these points were excluded from the analysis). The state of a point is given by the species (or bare rock) that occupies it; thus there are 15 possible states in our model. Table 1 shows the number of sampled points in each state and the abbreviations used to identify each state.

Maximum likelihood estimates of transition probabilities were obtained by creating a contingency table in which the $i, j$ entry, $n_{i j}$, gives the number of points that were in state $j$ at time $t$ and state $i$ at $t+1$. The transition probabilities, $p_{i j}$, were estimated as

$$
\widehat{p_{i j}}=\frac{n_{i j}}{\sum_{i} n_{i j}} .
$$

These estimates average over spatial and temporal variability to produce the best single estimate of a homogenous Markov chain for the subtidal community. The resulting transition matrix is shown in table 2 .

We will extract three levels of information from the model:

Species properties. These describe aspects of the biology of an individual species in the context of its community. For example, the probability that an empty point is colonized by the bryozoan Crisia eburnea is .32, while the corresponding probability for the polychaete Spirorbis spirorbis is only .01. Crisia is one of the most abundant species in the equilibrium community; Spirorbis is one of the rarest. We will use such comparisons to see to what extent species-specific properties are correlated with equilibrium abundance.

Community properties. These describe properties of the entire community rather than those of individual species. Some of them are obtained by averaging species properties over the equilibrium community composition. For example, the average time before a randomly selected point in the subtidal community changes state (its turnover time) is 3.4 years. We can compare such community properties with the corresponding properties of other communities.

Perturbation properties. These describe how changes in either the transition structure or the species pool would affect the resulting community. There are many such questions; we will focus on a small subset concerned with the diversity and evenness of the community.

\section{Successional Analysis}

\section{Stationary Community Structure}

We begin by using $\mathbf{P}$ to calculate the stationary, or equilibrium, community structure. If the transition matrix $\mathbf{P}$ is primitive (i.e., if $\mathbf{P}^{k}>0$ for some finite $k \geq 1$ ), then a community described by equation (2) will asymptotically approach an equilibrium, or stationary, probability distribution from any initial condition. This distribution is given by the dominant eigenvector of $\mathbf{P}$, normalized to sum to 1 ; we denote this eigenvector as $\mathbf{w}$. Many authors have focused on the stationary distribution as a prediction of asymptotic community composition (e.g., Waggoner and Stephens 1970; Usher 1979; Rego et al. 1993; Gibson et al. 1997; Wootton 2001b, 2001c).

Figure 1 shows the stationary structure of the subtidal community, with the biological species listed in decreasing order of abundance and bare rock at the end. By listing the species this way, correlations of species properties with abundance will be clearly visible in subsequent figures. The dominant species are the encrusting sponge Hymedesmia sp. 1, which occupies $34 \%$ of the substrate, and the erect bryozoan Crisia eburnea, which occupies $26 \%$ of the substrate. The remaining 12 species occupy $33.5 \%$ of the substrate, with frequencies ranging from $7.6 \%$ (Myxilla fimbriata) down to $0.5 \%$ (Spirorbis spirorbis). About $8.0 \%$ of the substrate is unoccupied.

The stationary structure computed from $\mathbf{P}$ agrees remarkably well with the observed structure of the subtidal community (fig. 2), explaining $98 \%$ of the variance in 
Table 2: Estimated transition matrix $\mathbf{P}$ for the subtidal community

\begin{tabular}{|c|c|c|c|c|c|c|c|c|c|c|c|c|c|c|c|}
\hline & HY1 & CRI & MXY & MYC & FIL & URT & ASC & APL & HY2 & IDM & COR & MET & PAR & SPI & BR \\
\hline HY1 & .771 & .145 & .052 & .017 & .117 & .009 & .241 & .199 & .056 & .309 & .056 & .025 & .321 & .158 & .101 \\
\hline CRI & .102 & 609 & .061 & .054 & .218 & .024 & .223 & .235 & .147 & .228 & .222 & .068 & .179 & .448 & .320 \\
\hline MXY & .017 & .031 & .710 & .006 & .035 & .012 & .051 & .038 & .026 & .031 & .028 & .018 & .023 & .018 & .025 \\
\hline MYC & .004 & .011 & .004 & .839 & .004 & .000 & .016 & .018 & .011 & .010 & .008 & .030 & .000 & .018 & .009 \\
\hline IL & .015 & .028 & .020 & .005 & .404 & .016 & .080 & .089 & .020 & .027 & .036 & .016 & .063 & .085 & .062 \\
\hline URT & .001 & .005 & .004 & .000 & .008 & .863 & .024 & .007 & .006 & .006 & .000 & .000 & .000 & .006 & .005 \\
\hline ASC & .018 & .022 & .008 & .004 & .033 & .001 & 105 & .044 & .011 & .042 & .025 & .010 & .030 & .030 & .048 \\
\hline APL & .012 & .025 & .008 & .006 & .032 & .007 & .041 & .154 & .026 & .031 & .020 & .016 & .020 & .018 & .034 \\
\hline HY2 & .002 & .011 & .025 & .008 & .013 & .016 & .014 & .015 & .586 & .010 & .007 & .004 & .003 & .018 & .013 \\
\hline IDM & .014 & .015 & .003 & .004 & .007 & .003 & .033 & .027 & .021 & .165 & .007 & .003 & .020 & .030 & .031 \\
\hline COR & .003 & .012 & .005 & .006 & .006 & .004 & .025 & .016 & .006 & .013 & .507 & .001 & .017 & .006 & .017 \\
\hline MET & .002 & .008 & .007 & .011 & .005 & .007 & .005 & .020 & .005 & .008 & .002 & .537 & .000 & .006 & .017 \\
\hline PAR & .005 & .005 & .002 & .000 & .006 & .000 & .014 & .009 & .001 & .012 & .005 & .003 & .248 & .000 & .011 \\
\hline SPI & .003 & .004 & .008 & .003 & .005 & .000 & .012 & .009 & .005 & .006 & .003 & .003 & .000 & .030 & .013 \\
\hline BR & .029 & .069 & .084 & .036 & .108 & .036 & .115 & .122 & .074 & .104 & .076 & .266 & .076 & .127 & .294 \\
\hline
\end{tabular}

Note: Species are ordered in decreasing order of abundance in the stationary community. Diagonal entries are in boldface type for reference.

species abundance $(r=0.997, P<.0005$ by a nonparametric randomization test). ${ }^{1}$

\section{Successional Transitions}

The entries of $\mathbf{P}$ describe the processes that determine succession: colonization, disturbance, replacement, and persistence. These can be quantified for individual species (column $j$ of $\mathbf{P}$ gives the probability of transitions from species $j$; row $i$ of $\mathbf{P}$ gives the probability of transitions to species $i$ ) and for the entire community. For an individual species:

Colonization. Colonization occurs when a species occupies a previously unoccupied point; it results from recruitment of larvae onto bare substrate or growth of individuals into unoccupied space. In this article, we will denote bare rock as state $s$ (the last state in our list). Then

$$
P(\text { colonization by species } j)=p_{j s} \text {. }
$$

Disturbance. Disturbance occurs when an occupied point at time $t$ becomes unoccupied at $t+1$. It results from either biological processes (e.g., disease, predation) or physical disturbance such as swells generated by severe winter storms (Witman 1987); its probability is

$$
P(\text { disturbance of species } j)=p_{s j} \text {. }
$$

\footnotetext{
${ }^{1}$ It may seem that figure 2 compares predictions with the same data used to make those predictions and that the result would be an artifactual agreement between the two (cf. Facelli and Pickett 1990; Wootton 2001a). This is not true because the estimation procedure and the comparison of structure use different, statistically independent parts of the data set. See Hill et al. (2002) for details.
}

Replacement. Replacement occurs when a point occupied by one species at time $t$ is occupied by a different species at time $t+1$. It results when a nearby species overgrows an inferior competitor (Russ 1982; Sebens 1986); its probability is

$$
\begin{aligned}
P(\text { replacement of species } j) & =1-p_{j j}-p_{s j} \\
j & =1, \ldots, s-1 .
\end{aligned}
$$

Instead of considering the replacement of a species, we can focus on replacement by a species. Row $i$ of $\mathbf{P}$ contains the probabilities that species $i$ replaces the other species in the community. The average of these probabilities is

$$
P(\text { replacement by species } i)=\frac{1}{s-2} \sum_{j \neq i, s} p_{i j} \text {, }
$$

where $p_{i i}$ and $p_{i s}$ are excluded from the summation because they represent persistence and colonization, not species replacements.

We will see later that "replacement of" and "replacement by" provide complementary pictures of how a species interacts with other species in the community.

Persistence. Persistence occurs when a point occupied by a species at time $t$ is still occupied by that species at $t+1$ :

$$
P(\text { persistence of species } j)=p_{j j} \text {. }
$$

Figure 3 shows these probabilities for each species. Comparison with figure 1 shows that dominance in the subtidal community is associated with a low probability of disturbance, high probabilities of colonization and per- 


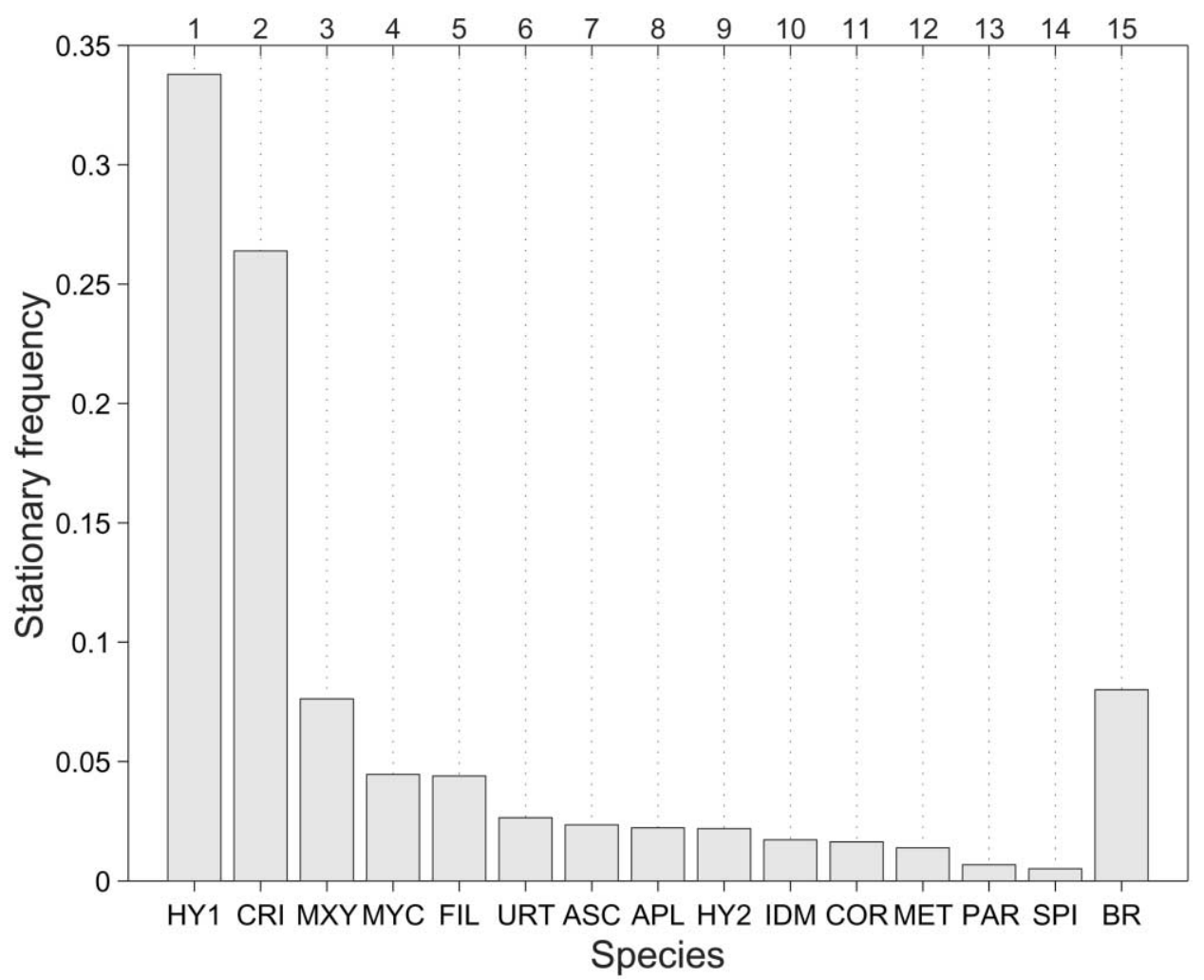

Figure 1: Stationary distribution of species frequencies in the subtidal community. Species are displayed in decreasing order of abundance, with bare rock $(B R)$ at the end.

sistence, a high probability of replacing other species ("replacement by"), and a low probability of being replaced by other species ("replacement of"). The positive correlations of dominance with colonization and the probability of "replacement by" are statistically significant (table 3).

Successional transitions can also be examined at the level of the community:

Colonization. The probability that an empty point is colonized by some species is

$$
\begin{aligned}
P(\text { colonization }) & =\sum_{i \neq s} p_{i s}=1-p_{s s} \\
& =0.71 .
\end{aligned}
$$

Disturbance. The probability that an occupied point, randomly selected from the stationary community, is disturbed between $t$ and $t+1$ is the average over the stationary distribution of the species-specific disturbance probabilities:

$$
\begin{aligned}
P(\text { disturbance }) & =\frac{\sum_{j \neq s} w_{j} p_{s j}}{\sum_{j \neq s} w_{j}} \\
& =0.06 .
\end{aligned}
$$

Replacement. The probability that a point, randomly selected from the stationary community, is replaced by a different species is the average over the stationary distribution of the probability of replacement:

$$
\begin{aligned}
P(\text { replacement of }) & =\frac{\sum_{i \neq s} w_{i}\left(1-p_{i i}-p_{s i}\right)}{\sum_{i \neq s} w_{i}} \\
& =0.29 .
\end{aligned}
$$

Equation (7) gives the average probability of replacement by species $i$. The average of this quantity over the stationary distribution gives

$$
\begin{aligned}
P(\text { replacement by }) & =\frac{\frac{1}{s-2} \sum_{i \neq s} w_{i} \sum_{j \neq i, s} p_{i j}}{\sum_{i \neq s} w_{i}} \\
& =0.10 .
\end{aligned}
$$

Note that the choice to exclude bare rock (state $s$ ) from the averages in equations (11) and (13) is a choice of 

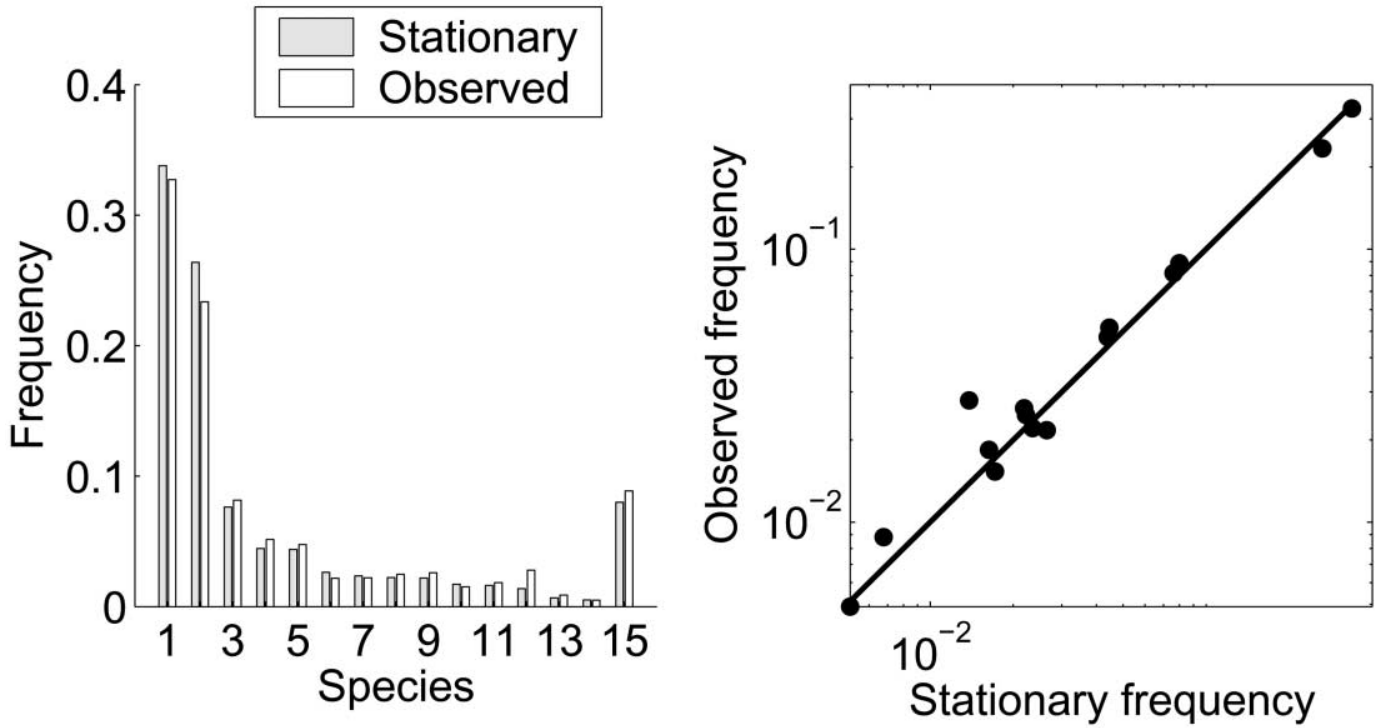

Figure 2: Left, Stationary distribution and the observed frequency distribution of species in the subtidal community. Species are displayed in decreasing order of abundance in the stationary distribution and in the order shown in figure 1. Right, Observed species frequencies as a function of the frequencies in the stationary distribution. The correlation between the observed and stationary distribution is $r=0.9838 ; P<.0005$ by a nonparametric permutation test.

interpretation; most ecologists would be concerned with colonization, disturbance, and replacement of biological species only, but there is no reason that comparable averages could not be calculated including bare rock or for other subsets of the community (e.g., all sponges).

In the subtidal community, the rate of disturbance is low (0.06), the colonization rate is high (0.71), and the replacement rate is intermediate (0.29). In "A Comparative Analysis of Benthic Communities," we will compare these values to other communities.

\section{Successional Dynamics}

Turnover Rates. The turnover rate measures the rate at which points change state and is thus one measure of the rate of successional change. The turnover rate of species $i$ is the probability that a point in state $i$ changes state between $t$ and $t+1$; it is

$$
\mathcal{T}_{i}=\left(1-p_{i i}\right)
$$

The inverse of the turnover rate gives the expected turnover time of species $i$ :

$$
E(\text { turnover time })=\tau_{i}=\frac{1}{\mathcal{T}_{i}}
$$

Figure 4 shows these turnover times. Most are short $(\leq 2$ years); the longest are 6.2 and 7.3 years. There is a positive but nonsignificant correlation of turnover time with dominance in the stationary community (table 3 ).

The mean turnover time of a point in the stationary community is the average over the stationary distribution of the $\tau_{i}$ :

$$
\bar{\tau}=\sum_{i=1}^{s} \frac{w_{i}}{\mathcal{T}_{i}}
$$

This includes the turnover time of bare rock (state $s$ ). Calculating the mean over only occupied points produces the biotic turnover time:

$$
\bar{\tau}_{\text {bio }}=\frac{\sum_{i \neq s} \frac{w_{i}}{\mathcal{T}_{i}}}{\sum_{i \neq s} w_{i}} .
$$

In the subtidal community, $\bar{\tau}_{\text {bio }}=3.4$ years.

Recurrence Times. A point in any state will eventually leave that state and then return to it at some later time. The Smoluchowski recurrence time of state $i$ is the average time elapsing between a point leaving state $i$ and then returning to it again. It is given by 

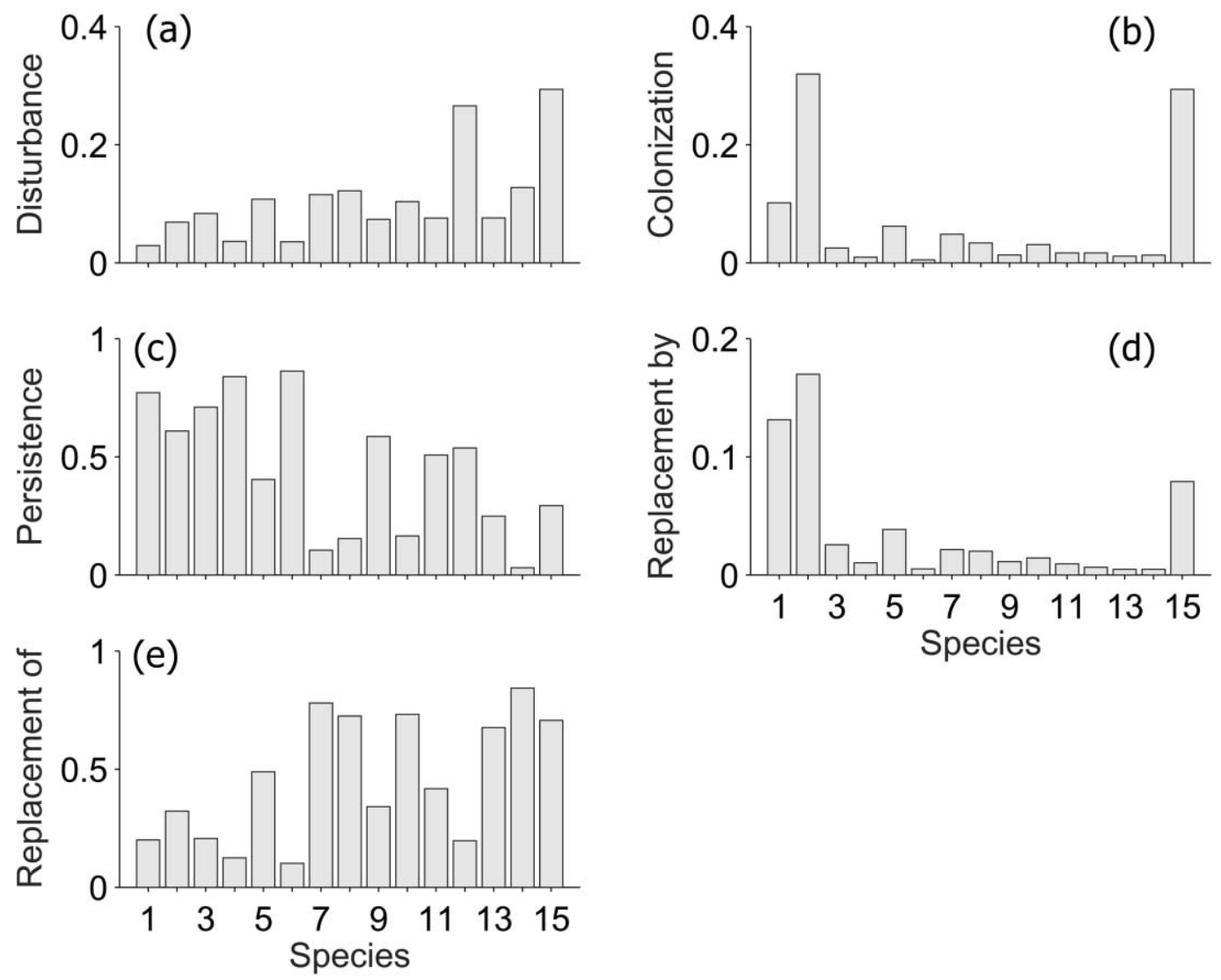

Figure 3: Species-specific transition probabilities from the community transition matrix $\mathbf{P}$ for the subtidal community. Species are displayed in decreasing order of abundance in the stationary distribution, in the order shown in figure 1. $a$, Probability that the species is disturbed in one unit of time. $b$, Probability that the species colonizes a patch of bare rock in one unit of time. $c$, Probability that a species persists for one unit of time. $d$, Average probability of replacement of other species. $e$, Probability that the species is replaced by some other species.

$$
\theta_{i}=\frac{1-w_{i}}{w_{i}\left(1-p_{i i}\right)}
$$

(Iosifescu 1980). These recurrence times are highly variable among species in the subtidal community, ranging from 7.1 to 268.5 years (fig. 4). There is a significant negative correlation between $\theta_{i}$ and abundance in the stationary community $(r=-0.55, P=.026$; see table 3$)$; that is, the rarer a species is, the longer it takes for that species to reoccupy a point.

In the stationary community, the mean recurrence time is

$$
\bar{\theta}=\sum_{i} w_{i} \theta_{i}
$$

and the biotic mean recurrence time is

In the subtidal community, the mean recurrence time is $\bar{\theta}=36.2$ years, and the biotic mean recurrence time is $\bar{\theta}_{\text {bio }}=37.9$ years.

The recurrence time for bare rock is the mean time that a point remains occupied once it has been colonized and is thus the mean time between disturbances. In the subtidal community, this is $\theta_{s}=16.2$ years. Since the biotic mean turnover time $\overline{\mathcal{T}}_{\text {bio }}=3.4$ years, a point changes its biotic state an average of $16.2 / 3.4=4.8$ times between disturbances.

Entropy, Complexity, and Predictability of Succession. In their study of coral reef succession, Tanner et al. (1994) noted that between $88 \%$ and $91 \%$ of the entries of $\mathbf{P}$ were positive; they called this high proportion of nonzero en- 
Table 3: Correlations between species properties and species abundance in the stationary community structure

\begin{tabular}{lrc}
\hline Variable & \multicolumn{1}{c}{$r$} & $P$ \\
\hline Transition probabilities: & & \\
$\quad$ Disturbance & -.2605 & .3325 \\
Colonization & .6035 & .0150 \\
Persistence & .4203 & .1135 \\
Replacement of & -.3791 & .1590 \\
Replacement by & .9277 & .0010 \\
Dynamic properties: & & \\
Turnover time & .2416 & .3990 \\
Recurrence time & -.5525 & .0265 \\
Species entropy & -.3383 & .2070 \\
Perturbation properties; & & \\
$\quad$ elasticity of $H$ to: & & \\
Disturbance & .8063 & .0050 \\
Colonization & -.8922 & .0045 \\
Persistence & -.8474 & .0040 \\
Replacement of & .9075 & .0060 \\
Replacement by & -.9153 & .0025 \\
\hline
\end{tabular}

Note: $P$ values are for tests of the null hypothesis of no correlation between the property in question and abundance, computed with a randomization test. Correlations were calculated for 2,000 random permutations of the species property; $P$ is the proportion of the resulting 2,001 correlations greater than or equal in absolute magnitude to the observed correlation.

tries the "most remarkable aspect" of their transition matrices and defined it as a "complexity index." The subtidal community has a similarly high complexity index (95\%).

However, the complexity index provides only limited information on succession because it is insensitive to the magnitude of the transition probabilities. Consider the column of $\mathbf{P}$ giving the transition probabilities for species $j$. The complexity index does not distinguish between

$$
\mathbf{p}_{j}=\left(\begin{array}{c}
0.25 \\
0.25 \\
0.25 \\
0.25
\end{array}\right) \text { and } \quad \mathbf{p}_{j}=\left(\begin{array}{c}
0.01 \\
0.97 \\
0.01 \\
0.01
\end{array}\right)
$$

because both have a complexity index of 1.0. But in the first case, the fate of a point occupied by species $j$ is completely uncertain, while in the second it is almost certain that species $j$ will be replaced by species 2 .

An index that takes into account not only the proportion of nonzero entries but also their relative magnitudes is the entropy of $\mathbf{p}_{. j}$ :

$$
H\left(\mathbf{p}_{. j}\right)=-\sum_{i} p_{i j} \log p_{i j} \cdot
$$

The entropies of the two columns in equation (20) are 1.38 and 0.17 . Thus the entropy of a species is an inverse measure of the predictability of its successional changes.

The species entropies for the subtidal community range from 0.69 to 2.2 (the maximum possible value is $\log 15=2.7$; fig. 4). There is a negative but nonsignificant correlation between species entropy and dominance in the stationary community (table 3 ).

The entropy of the Markov chain as a whole (e.g., Khinchin 1957; Reza 1961) is the average over the stationary distribution of the entropies of the columns of $\mathbf{P}$ :

$$
H(\mathbf{P})=-\sum_{j=1}^{s} w_{j} \sum_{i=1}^{s} p_{i j} \log p_{i j} .
$$

The quantity $H(\mathbf{P})$ gives the uncertainty in the fate of a point randomly selected from the stationary community. If $H(\mathbf{P})=0$, the state of a point in the next time step is completely known (i.e., the uncertainty is 0 ), and succession is completely deterministic. The maximum uncertainty occurs when $H(\mathbf{P})=H_{\max }(\mathbf{P})=\log (1 / s)$, in which case the fate of a point is completely unpredictable. Since $H_{\max }(\mathbf{P})$ depends on $s$, we can calculate the normalized entropy of $\mathbf{P}$ as

$$
H_{r}(\mathbf{P})=\frac{H(\mathbf{P})}{H_{\max }(\mathbf{P})} .
$$

The normalized entropy of the subtidal transition matrix is $H_{r}(\mathbf{P})=0.49$. This community is poised halfway between predictability and unpredictability.

\section{Community Convergence}

The rate of convergence to the stationary distribution can be measured in several ways (Cohen et al. 1993; Rosenthal 1995). One is the damping ratio

$$
\rho=\frac{\lambda_{1}}{\left|\lambda_{2}\right|},
$$

where $\lambda_{1}$ and $\lambda_{2}$ are the largest and second-largest eigenvalues of $\mathbf{P}$ (because $\mathbf{P}$ is stochastic, $\lambda_{1}=1$ ). Community structure converges to the equilibrium, in the long run, at least as fast as $\exp (-t \log \rho)$ (Caswell 2001), so $\rho$ provides a lower bound on the convergence rate. The closer the second eigenvalue is in magnitude to the first, the slower the rate of convergence. The half-life of a perturbation is given by $\log 2 / \log \rho$. The damping ratio has been applied to succession models by Tanner et al. (1994) and Wootton (2001c).

An alternative measure of convergence rate, not re- 

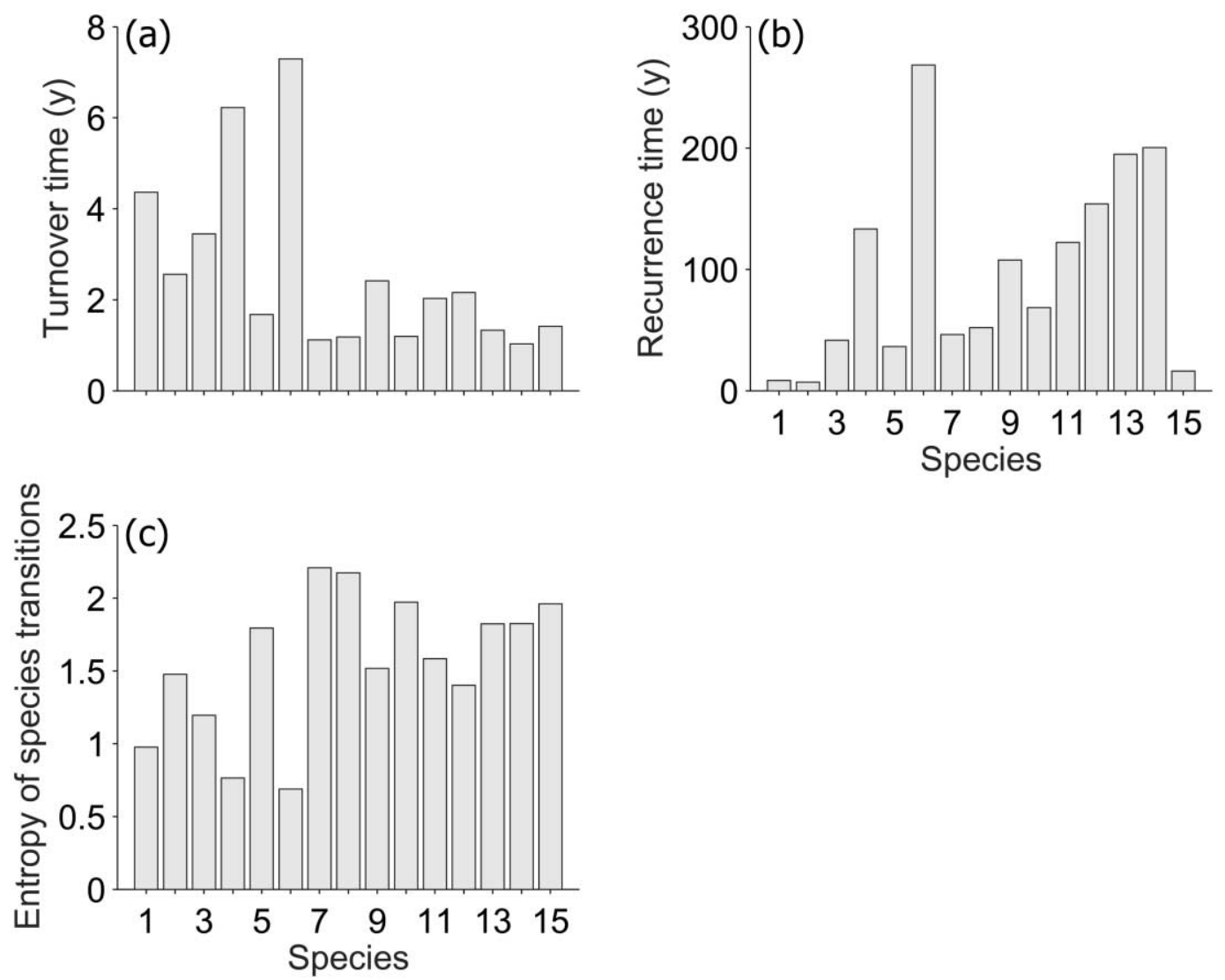

Figure 4: Species-specific dynamic properties of the subtidal community. Species are displayed in decreasing order of abundance in the stationary distribution, in the order shown in figure 1. $a$, Turnover time (years). $b$, Smoluchowski recurrence time (years). $c$, Entropy of the column in the transition matrix $\mathbf{P}$ corresponding to each species.

stricted to asymptotic conditions, is provided by Dobrushin's coefficient of ergodicity $\bar{\alpha}(\mathbf{P})$ (Dobrushin 1956a, $1956 b$ ). Imagine two copies of the same community starting from initial states $\mathbf{x}_{1}$ and $\mathbf{x}_{2}$. After one iteration the new states $\mathbf{P} \mathbf{x}_{1}$ and $\mathbf{P} \mathbf{x}_{2}$ will be closer together than $\mathbf{x}_{1}$ and $\mathbf{x}_{2}$ were, and the amount of contraction is given by

$$
\frac{\left\|\mathbf{P} \mathbf{x}_{1}-\mathbf{P} \mathbf{x}_{2}\right\|}{\left\|\mathbf{x}_{1}-\mathbf{x}_{2}\right\|}
$$

where $\|\cdot\|$ denotes the 1-norm. Dobrushin's coefficient,

$$
\bar{\alpha}(\mathbf{P})=\sup _{\mathbf{x}_{1}, \mathbf{x}_{2}}\left(\frac{\left\|\mathbf{P x}_{1}-\mathbf{P} \mathbf{x}_{2}\right\|}{\left\|\mathbf{x}_{1}-\mathbf{x}_{2}\right\|}\right),
$$

gives an upper bound on the contraction, and hence $-\log \bar{\alpha}(\mathbf{P})$ gives a lower bound on the convergence rate, comparable to $\log \rho$. Dobrushin's coefficient is calculated from $\mathbf{P}$ as

$$
\bar{\alpha}(\mathbf{P})=\frac{1}{2} \max _{j, k}\left\|\mathbf{p}_{j}-\mathbf{p}_{. k}\right\|,
$$

where $\mathbf{p}_{. j}$ is column $j$ of $\mathbf{P}$.

Unlike the damping ratio, Dobrushin's coefficient reveals an important connection between the transition probabilities and the convergence rate. Dobrushin's coefficient depends on the differences among the columns of $\mathbf{P}$, each of which is the probability distribution of the fate of a point occupied by one of the species. If all the species behave identically, then all the columns of $\mathbf{P}$ are identical, $\bar{\alpha}(\mathbf{P})=0$, and the community converges to its stationary distribution in a single iteration. At the other extreme, if the species have completely different fates, then $\bar{\alpha}(\mathbf{P})=1$ and the only possible stochastic matrix is one that cycles deterministically among the states, and the community never converges. The convergence rate of the community is thus inversely related to the ecological differences among the species. 
In the subtidal community, $\rho=1.16$, implying a convergence rate of $\log \rho=0.15$ (i.e., $\sim 15 \%$ /year) and a halflife of 4.7 years. Dobrushin's coefficient is $\bar{\alpha}=0.89$, implying a short-term convergence rate of $-\log \bar{\alpha}=0.12$ ( 12\%/year).

\section{Perturbation Analysis}

Perturbation analysis plays an essential role in demographic analysis (Tuljapurkar and Caswell 1997; Caswell 2001) and can be equally valuable in community modeling. In this section we examine the elasticity of species diversity to changes in the $p_{i j}$ and the effects on evenness of eliminating a species from the community. Many other perturbation analyses are possible.

\section{Changes in Transition Probabilities}

Eigenvector sensitivity analysis permits the calculation of the effects on diversity of perturbations to $\mathbf{P}$. The stationary community composition is given by the dominant eigenvector $\mathbf{w}_{1}$ of $\mathbf{P}$, scaled to sum to 1 . The sensitivity of this scaled eigenvector to changes in the transition probability $p_{i j}$ is

$$
\frac{\partial \frac{\mathbf{w}_{1}}{\left\|\mathbf{w}_{1}\right\|}}{\partial p_{i j}}=\frac{\partial \mathbf{w}_{1}}{\partial p_{i j}}-\mathbf{w}_{1} \sum_{\mathrm{k}} \frac{\partial w_{k}}{\partial p_{i j}},
$$

where

$$
\frac{\partial \mathbf{w}_{1}}{\partial p_{i j}}=w_{j}^{(1)} \sum_{m \neq 1}^{s} \frac{v_{i}^{(m)}}{\lambda_{1}-\lambda_{m}} \mathbf{w}_{m} .
$$

Here, $w_{j}^{(1)}$ is the $j$ th element of $\mathbf{w}_{1}, v_{i}^{(m)}$ is element $i$ of the left eigenvector $\mathbf{v}_{m}$, and $\lambda_{m}$ is the $m$ th eigenvalue (see Caswell 2001, section 9.4). However, equation (29) cannot be directly applied to Markov chain models (as attempted by Tanner et al. [1994]) because in the context of Markov chains, the only perturbations of interest are those that preserve the column sums of $\mathbf{P}$, which must equal 1 . Thus any change in $p_{i j}$ must be accompanied by compensating changes in the other entries of column $j$. The resulting total derivative is

$$
\frac{d \frac{\mathbf{w}_{1}}{\left\|\mathbf{w}_{1}\right\|}}{d p_{i j}}=\frac{\partial \frac{\mathbf{w}_{1}}{\left\|\mathbf{w}_{1}\right\|}}{\partial p_{i j}}+\sum_{m \neq i}^{s} \frac{\partial \frac{\mathbf{w}_{1}}{\left\|\mathbf{w}_{1}\right\|}}{\partial p_{m j}} \frac{\partial p_{m j}}{\partial p_{i j}}
$$

(Caswell 2001). In equation (30), the derivatives $\partial p_{m j} / \partial p_{i j}$ for $m \neq i$ are determined so that the change in $p_{i j}$ is compensated for by changes in the other entries in column $j$ of $\mathbf{P}$. While several compensation patterns are possible (see Caswell 2001 for details), here we use proportional compensation:

$$
\frac{\partial p_{m j}}{\partial p_{i j}}=\frac{-p_{m j}}{1-p_{i j}} \quad m \neq i
$$

in which the change in $p_{i j}$ is distributed over the other entries in the jth column proportional to their value. Other compensation methods give qualitatively similar results.

We use the eigenvector sensitivities (eq. [30]) to compute the sensitivity of the Shannon-Wiener diversity index. This index is usually calculated only over the biotic states, that is, excluding bare rock. The stationary proportions of the biotic states are

$$
w_{i}^{\prime}=\frac{w_{i}}{1-w_{s}} \quad i \neq s,
$$

and the Shannon-Wiener index is the entropy of $\mathbf{w}^{\prime}$ :

$$
H\left(\mathbf{w}^{\prime}\right)=-\sum_{i} w_{i}^{\prime} \log w_{i}^{\prime}
$$

The sensitivity of $\mathrm{H}\left(\mathbf{w}^{\prime}\right)$ to changes in $p_{i j}$ is

$$
\frac{d H}{d p_{i j}}=-\sum_{i}\left(1+\log w_{i}^{\prime}\right) \frac{d w_{i}^{\prime}}{d p_{i j}},
$$

where

$$
\frac{d w_{i}^{\prime}}{d p_{i j}}=\frac{\left(1-w_{s}\right) d w_{i} / d p_{i j}+\left(w_{i}\right) d w_{s} / d p_{i j}}{\left(1-w_{s}\right)^{2}}
$$

and $d w_{m} / d p_{i j}$ is the $m$ th element of the vector given by equation (30).

We will focus on the elasticities, or proportional sensitivities of $H$, given by

$$
\frac{p_{i j}}{H} \frac{\partial H}{\partial p_{i j}}
$$

The elasticity of $H$ to proportional changes in a set of transitions (e.g., the probabilities that a species is replaced by another) is simply the sum of the elasticities of $H$ to those transitions. For example, the elasticity of $H$ to the probability that species $j$ is replaced by some other species is

$$
\sum_{i \neq j, s} \frac{p_{i j}}{H} \frac{\partial H}{\partial p_{i j}} .
$$

Figure 5 shows the elasticities of $H$ to changes in the 

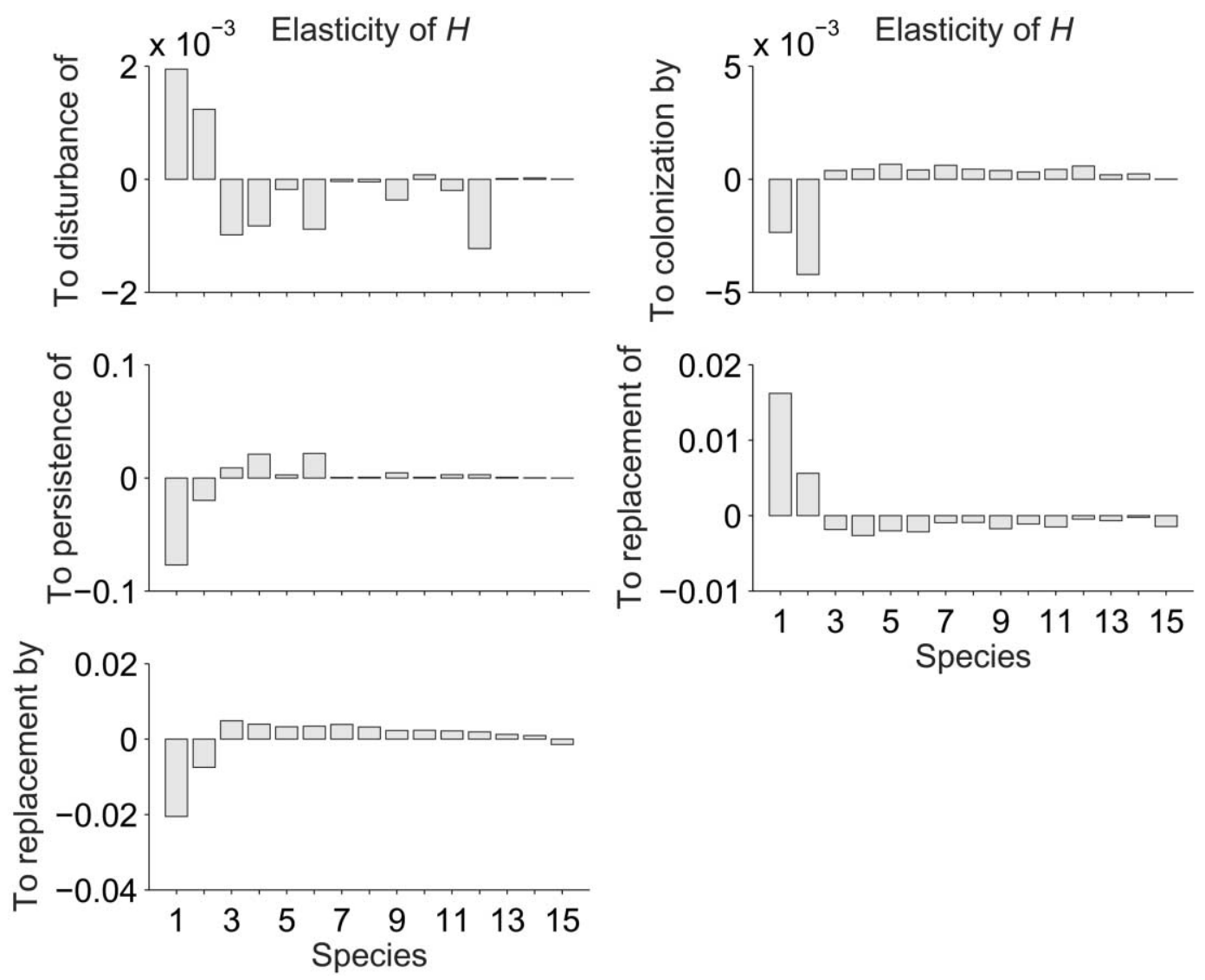

Figure 5: Elasticity of species diversity $H$ to changes in transition probabilities of each species in the subtidal community. Species are displayed in decreasing order of abundance in the stationary distribution, in the order shown in figure 1.

probability of disturbance of, colonization by, persistence of, replacement of, and replacement by each species in the community. These elasticities are significantly correlated with species dominance in the stationary community (table 3). Increasing the disturbance of or the replacement of the dominant species or reducing the colonization of, persistence of, or replacement by the dominant species will all increase diversity. The converse is true for the rare species, although the effects of such changes are generally small. The scales on the axes in figure 5 are different. By far the largest elasticities are those of $H$ to changes in persistence probability. Thus, in the subtidal community, the most dramatic way to change diversity would be to change the ability of species to hold onto occupied space.

\section{Species Removal}

Species removal experiments are an important way of characterizing the effects of extinction on diversity and community resilience (Paine 1974, 1992; Menge et al. 1994).
Species whose removal produces a dramatic effect are termed strong interactors (MacArthur 1972; Paine 1980) or keystone species (Paine 1966) if their effect is disproportional to their abundance. The removal of such a species can lead to dramatic changes in community composition (Hughes 1994).

Here we use the Markov chain model to examine effects of species removal on diversity of the stationary community. Because species removal automatically changes species richness, we measure diversity by the evenness:

$$
J=\frac{H\left(\mathbf{w}^{\prime}\right)}{\log s}
$$

$J$ reaches its maximum value of 1 when all species are in the community are equally abundant (Lloyd and Ghelardi 1964).

To simulate the removal of species $i$, we set row $i$ and column $i$ of $\mathbf{P}$ to 0 , renormalize the other columns to sum to 1 , and then remove row $i$ and column $i$. Let $\mathbf{P}_{i}$ represent 
the transition matrix when state $i$ is removed, $\mathbf{w}^{(i)}$ its stationary distribution, and $J_{i}$ the corresponding evenness. The proportional change in evenness is

$$
\Delta J_{i}=\frac{J_{i}-J}{J} .
$$

Figure 6 shows $\Delta J_{i}$ for all species; the effects are relatively small, and there is no evidence of a keystone species in the intertidal community by this measure. The removal of the sponge Myxilla or the bryozoan Crisia would produce the largest declines in evenness $(9.7 \%$ and $7.8 \%$, respectively). Removing the most abundant species $\mathrm{Hy}$ medesmia sp. 1 would increase evenness, but removing any of the subdominant species would reduce evenness. This suggests that when present, the subdominant species increase evenness by slowing dominance by Hymedesmia.

\section{Discussion}

Markov chains are a powerful framework for the study of succession. They have permitted us to characterize the asymptotic structure, successional rates, and transition processes in the rocky subtidal community. Using pertur- bation analyses, we have documented the effects on succession of changes in transition probabilities. In such calculations, it is important to restrict perturbations to those that maintain the column-stochasticity of the transition matrix. When we do so, we find that the elasticities of diversity are highest to changes in the persistence of the dominant and subdominant species. Changes in the probabilities of disturbance and colonization have much smaller effects on diversity. Removal of subdominant species reduces the evenness of the remaining community, which suggests the existence of second-order effects of those subdominants in reducing dominance by Hymedesmium.

These results can be thought of as a kind of quantitative fingerprint of a community in terms of its successional processes and can be compared across systems. As an example, we compare our results to two other marine communities by analyzing the matrices reported by Tanner et al. (1994) for coral reefs and Wootton (2001c) for a rocky intertidal community.

\section{A Comparative Analysis of Benthic Communities}

Three studies have now used Markov chains to describe succession in marine benthic communities: our analysis

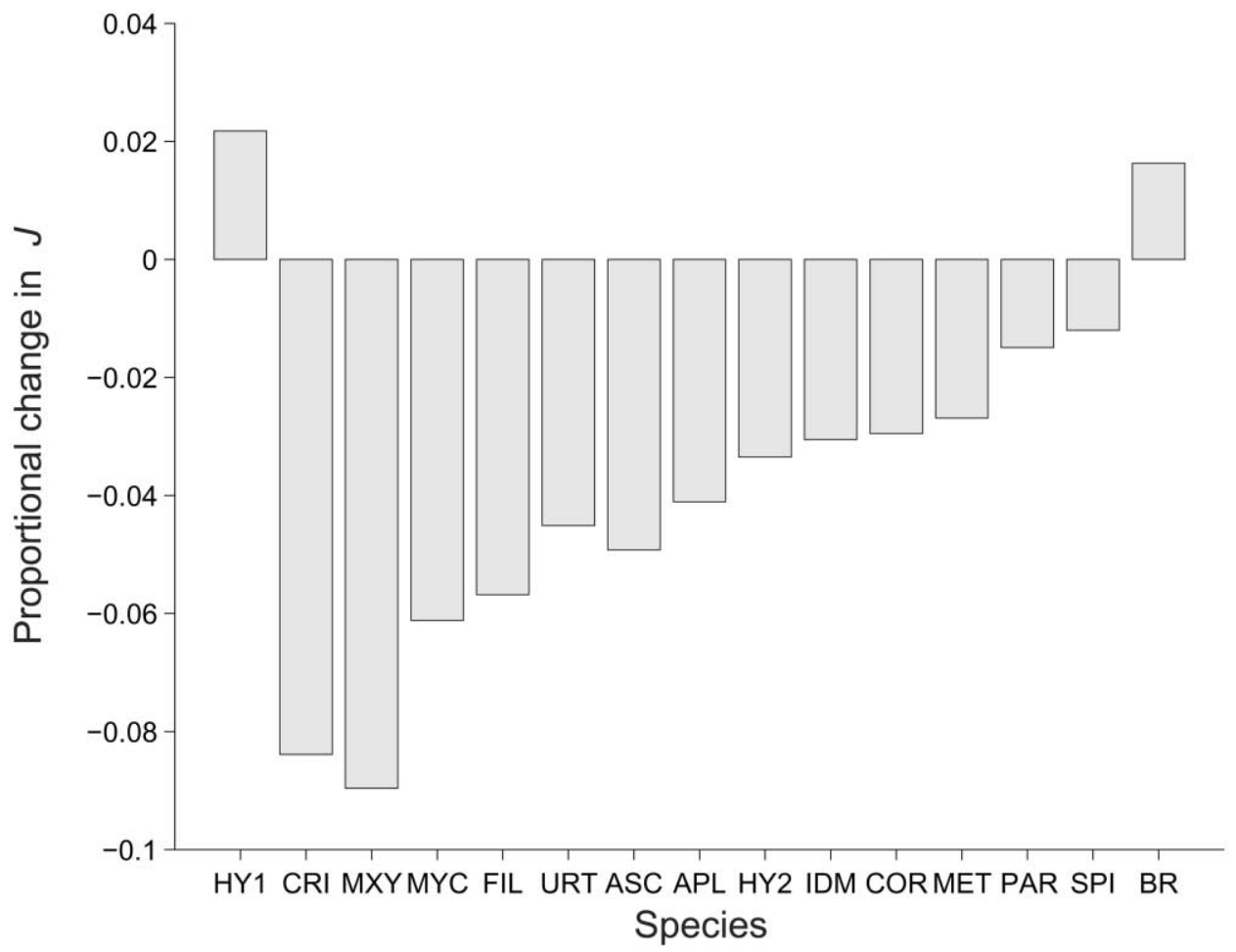

Figure 6: Proportional change in the biotic evenness $J$ resulting from eliminating species. Species are displayed in decreasing order of abundance in the stationary distribution, in the order shown in figure 1. 
of the subtidal community; a study by Tanner et al. (1994) of three coral reef communities on Heron Island, Australia; and a study by Wootton $(2001 b, 2001 c)$ of a rocky intertidal community on the coast of Washington. Comparisons of successional properties across these communities are informative (table 4).

Transition Probabilities. There are distinct differences in successional processes among the communities. Disturbance rates are an order of magnitude higher in the coral reef communities than in the subtidal or intertidal communities. In contrast, colonization rates are highest in the subtidal and intertidal communities and lowest in the coral reefs. The combination of a high disturbance rate and a low colonization rate explains why over $50 \%$ of the coral substrate is unoccupied at equilibrium, compared with $8 \%$ for the subtidal communities and $2 \%$ for the intertidal communities. Rates of replacement (averaged over the biotic components of the community) are much lower in the coral reefs than in the subtidal or intertidal communities.

The entropy of succession (eq. [23]) is highest in the intertidal and subtidal communities and much lower in the coral reef. Thus the successional sequence of a point is most predictable in the coral reef and less predictable in the intertidal or subtidal communities.

Rates of Succession. Mean biotic turnover rates $\overline{\mathcal{T}}_{\text {bio }}$ do not differ much among the communities. The characteristic turnover times $\bar{\tau}_{\text {bio }}$ range from 3 to 5 years, shortest in the intertidal community and longest for coral reefs. The biotic recurrence times $\bar{\theta}_{\text {bio }}$ are shortest for the intertidal community (15 years) and longest for the coral reefs (38-121 years). The subtidal community is intermediate, with a recurrence time of 38 years.

The rates of succession for empty points (bare rock) reveal striking differences among the communities. The turnover rate of bare rock is highest in the intertidal community, where an empty point persists only for an average of 1.07 years. The subtidal community is not far behind, with a turnover time of 1.43 years. Empty space persists much longer in the coral reef, with turnover times of $3-$ 7 years.

The stationary distribution in the coral reef communities is dominated by open space, which is rare in the subtidal and intertidal communities. The biotic evenness is highest in the coral reef communities because, of the eight species present, none occupies more than $20 \%$ of the substrate at equilibrium. In contrast, in the intertidal community approximately two-thirds of the substrate is occupied by a single species (Mytilus californianus). In the subtidal community, the two most abundant species occupy $60 \%$ of the substrate.

The results in table 4 can be combined into a description of succession in these three communities:

Subtidal. A typical point in the subtidal is disturbed about every 16 years. It remains empty for about 1.4 years before being colonized. The species occupying the point after colonization change every 3.4 years. Once eliminated from a point, a species returns to the point, on average, in 38 years. A randomly selected point experiences about five species replacements before being disturbed again.

Table 4: Community properties calculated from transition matrices for three marine benthic communities

\begin{tabular}{|c|c|c|c|c|c|}
\hline & Subtidal & Intertidal & Coral reef EC & Coral reef EP & Coral reef $\mathrm{PC}$ \\
\hline Mean disturbance rate & .06 & .02 & .39 & .41 & .36 \\
\hline Mean colonization rate & .71 & .93 & .14 & .26 & .34 \\
\hline Mean persistence rate & .62 & .51 & .78 & .64 & .57 \\
\hline Replacement by (biotic) & .10 & .26 & .03 & .04 & .07 \\
\hline Replacement of (biotic) & .29 & .45 & .08 & .12 & .16 \\
\hline Proportion bare rock & .08 & .02 & .74 & .61 & .51 \\
\hline Normalized entropy & .49 & .52 & .34 & .25 & .21 \\
\hline Bio turnover rate & .36 & .47 & .48 & .52 & .52 \\
\hline Bio turnover time (years) & 3.4 & 2.9 & 4.8 & 5.2 & 3.3 \\
\hline Bio recurrence time (years) & 37.9 & 14.6 & 121.3 & 87.0 & 38.1 \\
\hline BR turnover rate & .70 & .93 & .14 & .26 & .34 \\
\hline BR turnover time (years) & 1.43 & 1.07 & 7.14 & 3.84 & 2.94 \\
\hline BR recurrence time (years) & 16.3 & 42.9 & 5.5 & 5.3 & 4.5 \\
\hline Number of turnovers between disturbance & 4.8 & 5.1 & 1.15 & 1.02 & 1.36 \\
\hline Dobrushin's coefficient & .89 & .61 & .66 & .84 & .65 \\
\hline Bio evenness & .71 & .56 & .82 & .83 & .77 \\
\hline
\end{tabular}

Note: Values for the intertidal community are calculated from matrices reported by Wootton (2001a) for a mussel bed community in Washington. Values for the coral reef communities are calculated from matrices reported by Tanner et al. (1994) in a study on Heron Island, Great Barrier Reef, for an exposed reef crest (EC), a protected reef crest (PC), and an exposed pool (EP). 
The subtidal community converges to a stationary distribution with $8 \%$ bare rock and a biotic evenness of $J_{\text {bio }}=0.71$. Deviations from this stationary state decay at a rate of at least $12 \%$ year.

Intertidal. A typical point in the intertidal community is disturbed about every 40 years. A disturbed point is colonized almost immediately; the turnover time of bare rock is only 1.07 years. The species occupying the point change about every 3 years; once replaced, it takes 15 years for a species to return to a point. A point experiences 15 species replacements before it is disturbed and returned to bare rock.

The intertidal community converges to a stationary distribution characterized by only $2 \%$ bare rock, at which the biotic states have an evenness of $J_{\text {bio }}=0.56$. Deviations from the stationary community decay at a rate of at least $39 \% /$ year.

Coral reefs. A typical point in the coral reef is disturbed every $4-5$ years. Colonization is slow; a typical point remains empty for 3-7 years. Once occupied, the species occupying the point change every 3-5 years. Because replacement rates are low, once a species leaves a point, it does not return for $40-120$ years. The point experiences only about one replacement before being disturbed again.

The coral reef community converges, at a rate of $17 \%$ $23 \% /$ year, to a stationary distribution with $65 \%-84 \%$ bare space and a biotic evenness of $0.77-0.82$.

These comparisons are influenced to an unknown degree by the standard practice of pooling species in constructing the transition matrix. In the coral reef study, 72 species of coral and nine species of algae were pooled into eight species groups (Tanner et al. 1994). In the intertidal community, about 30 species were pooled into 13 species groups (Wootton 2001c). We did not pool species into groups in the subtidal analysis. Methods now exist for carrying out such pooling according to objective criteria that minimize its effects on community dynamics (Hill 2000), the results of which will be presented in another article.

We urge the use of Markov chain models as a tool for comparative community analysis; such comparisons will become more valuable as additional measures of community transition matrices are reported.

\section{Acknowledgments}

The authors would like to thank G. Flierl, M. Fujiwara, M. Nuebert, and J. Pineda for their comments; J. Leichter and S. Genovese for their help in collecting photo quadrat data; and K. Sebens for collaborating on offshore cruises. Comments from two anonymous reviewers improved a previous version of the manuscript. This research was supported by National Science Foundation grants DEB-
9527400, OCE-981267, OCE-9302238, and OCE-0083976 and by the National Oceanic and Atmospheric Administration's National Undersea Research Program, University of Connecticut-Avery Point. Woods Hole Oceanographic Institution contribution 10188.

\section{Literature Cited}

Aaviksoo, K. 1995. Simulating vegetation dynamics and land use in a mire landscape using a Markov model. Landscape and Urban Planning 31:129-142.

Caswell, H. 2001. Matrix population models: construction, analysis, and interpretation. $2 \mathrm{~d}$ ed. Sinauer, Sunderland, Mass.

Caswell, H., and J. E. Cohen. 1991a. Communities in patchy environments: a model of disturbance, competition, and heterogeneity. Pages 97-122 in J. Kolasa and S. T. A. Pickett, eds. Ecological heterogeneity. Springer, New York.

1991b. Disturbance, interspecific interaction and diversity in metapopulations. Biological Journal of the Linnaean Society 42:193-218.

Caswell, H., and R. J. Etter. 1999. Cellular automaton models for competition in patchy environments: facilitation, inhibition, and tolerance. Journal of Mathematical Biology 61:625-649.

Cohen, J. E., Y. Iwasa, G. Rautu, M. B. Rusaki, E. Seneta, and G. Zbaganu. 1993. Relative entropy under mappings by stochastic matrices. Linear Algebra and Its Applications 179:211-235.

Connell, J. H., and R. O. Slatyer. 1977. Mechanisms of succession in natural communities and their roles in community stability and organization. American Naturalist 111:1119-1144.

Dobrushin, R. L. 1956a. Central limit theorem for nonstationary Markov chains. I. Theory of Probability and Its Applications 1:65-80.

. 1956b. Central limit theorem for nonstationary Markov chains. II. Theory of Probability and Its Applications 1:329-383.

Facelli, J. M., and S. T. A. Pickett. 1990. Markovian chains and the role of history in succession. Trends in Ecology \& Evolution 5:27-30.

Gibson, D. J., J. S. Ely, and P. B. Looney. 1997. A Markovian approach to modeling succession on a coastal barrier island following beach nourishment. Journal of Coastal Research 13:831-841.

Hill, M. F. 2000. Spatial models of metapopulations and benthic communities in patchy environments. Ph.D. dissertation. Massachusetts Institute of TechnologyWoods Hole Oceanographic Institution.

Hill, M. F., J. D Witman, and H. Caswell. 2002. Spatio- 
temporal variation in Markov chain models of subtidal community succession. Ecology Letters 5:665-675.

Horn, H. S. 1975. Markovian properties of forest succession. Pages 196-211 in M. L. Cody and J. M. Diamond, eds. Ecology and evolution of communities. Harvard University Press, Cambridge, Mass.

Hughes, T. P. 1994. Catastrophes, phase shifts, and largescale degradation of a Caribbean coral reef. Science 265: 1547-1551.

Iosifescu, M. 1980. Finite Markov processes and their applications. Wiley, New York.

Isagi, Y., and N. Nakagoshi. 1990. A Markov approach for describing post-fire succession of vegetation. Ecological Research 5:163-171.

Keough, M. J. 1984. Dynamics of the epifauna of the bivalve Pinna bicolor: interactions among recruitment, predation, and competition. Ecology 65:677-688.

Khinchin, A. A. 1957. Mathematical foundations of information theory. Dover, New York.

Leichter, J. J., and J. D. Witman. 1997. Water flow over subtidal rock walls: relationship to distribution and growth rate of sessile suspension feeders in the Gulf of Maine. Journal of Experimental Marine Biology and Ecology 209:293-307.

Lloyd, M., and R. J. Ghelardi. 1964. A table for calculating the equability component of species diversity. Journal of Animal Ecology 33:217-225.

MacArthur, R. H. 1972. Strong or weak interactions? Transactions of the Connecticut Academy of Arts and Science 44:177-188.

Masaki, T., W. Suzuki, K. Niiyama, S. Iida, H. Tanaka, and T. Nakashizuka. 1992. Community structure of a species-rich temperate forest, Ogawa Forest Reserve, central Japan. Vegetatio 98:97-111.

Menge, B. A., E. L. Berlow, C. A. Blanchette, S. A. Navarrete, and S. B. Yamada. 1994. The keystone species concept: variation in interaction strength in a rocky intertidal zone. Ecological Monographs 64:249-286.

Osman, R. W. 1982. Artificial substrates as ecological islands. Pages 71-114 in J. Cairns, ed. Artificial substrates. Ann Arbor Science, Ann Arbor, Mich.

Paine, R. T. 1966. Food web complexity and species diversity. American Naturalist 100:65-75.

- 1974. Intertidal community structure: experimental studies on the relationship between a dominant competitor and its principle predator. Oecologia (Berlin) 15:93-120.

. 1980. Food webs: linkage, interaction strength and community infrastructure. The third Tansley lecture. Journal of Animal Ecology. 49:667-685.

- 1992. Food-web analysis through field measurement of per capita interaction strengths. Nature 355: $73-75$.
Rego, F., J. Pereira, and L. Trabaud. 1993. Modeling community dynamics of a Quercus coccifera $\mathrm{L}$. garrigue in relation to fire using Markov chains. Ecological Modelling 66:251-260.

Reza, F. M. 1961. An introduction to information theory. McGraw-Hill, New York.

Rosenthal, J. S. 1995. Convergence rates for Markov chains. SIAM Review 37:387-405.

Runkle, J. R. 1981. Gap generation in some old growth forests of the eastern United States. Ecology 62:10411051.

Russ., G. R. 1982. Overgrowth in a marine epifaunal community: competition hierarchies and competitive networks. Oecologia (Berlin) 53:12-19.

Sebens, K. P. 1986. Spatial relationships among encrusting marine organisms in the New England subtidal zone. Ecological Monographs 56:73-96.

Sutherland, J. P., and R. H. Karlson. 1977. Development and stability of the fouling community at Beaufort, North Carolina. Ecological Monographs 47:425-446.

Tanner, J. E., T. P. Hughes, and J. H. Connell. 1994. Species coexistence, keystone species, and succession: a sensitivity analysis. Ecology 75:2204-2219.

- 1996. The role of history in community dynamics: a modeling approach. Ecology 77:108-117.

Tuljapurkar, S., and H. Caswell. 1997. Structured population models in marine, terrestrial and freshwater systems. Chapman \& Hall, New York.

Usher, M. B. 1979. Markovian approaches to ecological succession. Journal of Animal Ecology 48:413-426.

Waggoner, P. E., and G. R. Stephens. 1970. Transition probabilities for a forest. Nature 255:1160-1161.

Witman, J. D. 1985. Refuges, biological disturbance, and rocky subtidal community structure in New England. Ecological Monographs 55:421-445.

- 1987. Subtidal coexistence: storms, grazing mutualism, and the zonation of kelp and mussels. Ecological Monographs 57:167-187.

1992. Physical disturbance and community structure of exposed and protected reefs: a case study from St. John, USVI. American Zoologist 32:641-654.

Witman, J. D., and P. K. Dayton. 2001. Rocky subtidal communities. Pages 339-366 in M. D. Bertness, S. D. Gaines, and M. Hay, eds. Marine community ecology. Sinauer, Sunderland, Mass.

Witman, J. D., and K. P. Sebens. 1988. Benthic community structure at a subtidal rock pinnacle in the central Gulf of Maine. Pages 67-104 in I. Babb and M. DeLuca, eds. Benthic productivity and marine resources of the Gulf of Maine. National Undersea Research Program Report 88-3.

Wootton, J. T. 2001 a.. Causes of species diversity differ- 
ences: a comparative analysis of Markov models. Ecology Letters 4:46-56.

. 2001b. Local interactions predict large-scale pattern in empirically derived cellular automata. Nature 413:841-844.
. 2001c. Predictions in complex communities: analysis of empirically derived Markov models. Ecology 82: 580-598.

Associate Editor: William J. Boecklen 